\title{
Annotations
}

\section{Nuclear magnetic resonance imaging of the brain}

The clinical uses of nuclear magnetic resonance (NMR) imaging systems are currently being evaluated in at least a dozen centres worldwide. Early results from examination of the brain in adults have been very promising. High contrast is seen between grey and white matter, and unlike $x$-ray computed tomography (CT), bone artefact is not a problem in the posterior fossa. A variety of pulse sequences is available; some show anatomy well and others highlight abnormalities. Direct coronal and sagittal imaging is readily achieved and the technique is sensitive to a variety of pathological changes. ${ }^{12}$ Unlike CT there is no known hazard with NMR imaging. ${ }^{34}$ Although most examinations have been performed on adults, a few children have been studied and the technique may have future paediatric applications.

\section{Instrumentation}

All NMR machines are based on a large magnet which gives a uniform static magnetic field. Protons behave like tiny spinning bar magnets and the static magnetic field aligns these, producing a net proton magnetisation. Radio frequency magnetic pulses perpendicular to the static field are used to change the orientation of magnetisation, after which it recovers or relaxes back to its original position. This recovery is exponential and the time constants describing relaxation in the longitudinal

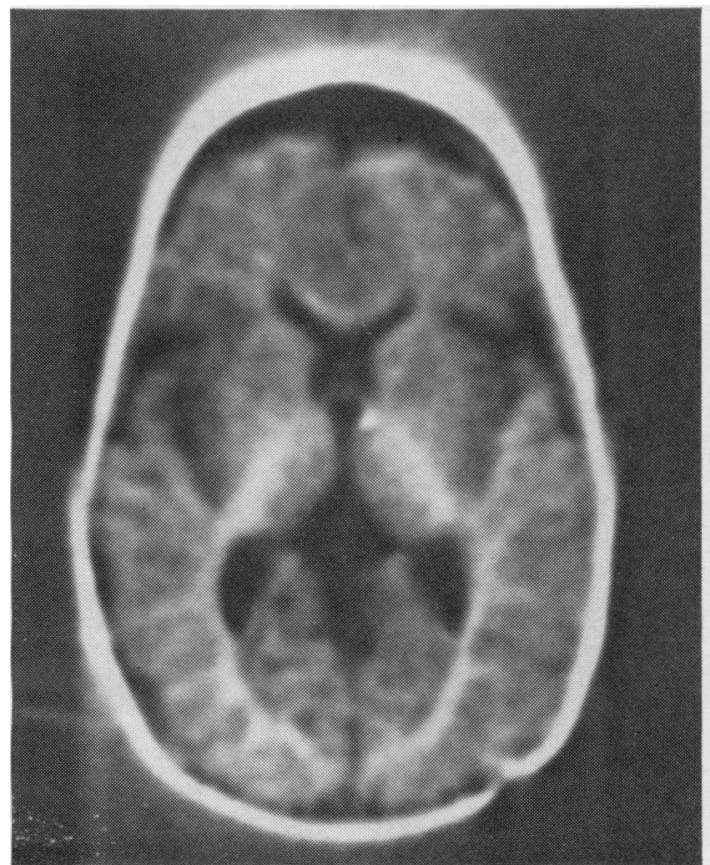

(a)

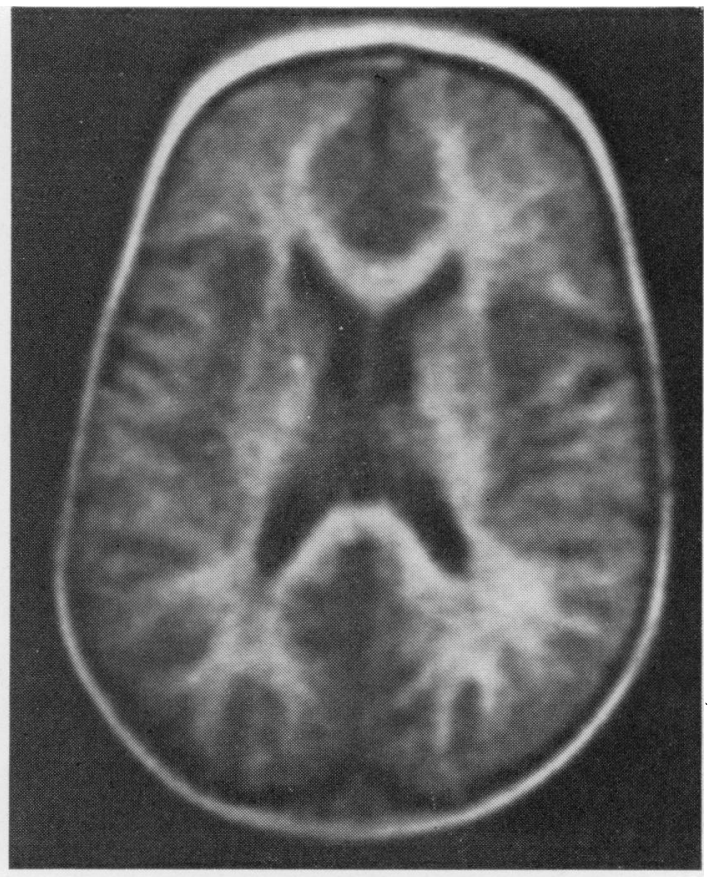

(b)

Fig. 1 Normal children aged 10 months (a) and 20 months $(b): T_{1}$ dependent scans. On both scans white matter appears white and grey matter grey. The posterior internal capsule and occipitothalmic radiation are seen at 10 months (a). By 20 months the forceps major, forceps minor, internal and external capsules are also visible (b). 
and transverse axes are known as $T_{1}$ and $T_{2}$ respectively. $T_{1}$ depends on the interaction of protons with surrounding nuclei and molecules, whereas $T_{2}$ depends on the interaction of protons with each other. Both $T_{1}$ and $T_{2}$ are sensitive indices of the local nuclear and molecular environment, and differences in the $T_{1}$ and $T_{2}$ of tissues are the main source of contrast in NMR images.

All NMR machines in use are research prototypes and many of these have been situated in factories or other clinically disadvantageous sites. Imaging has been a slow process, but the recent development of simultaneous multiple slice imaging promises greater speed in the future.

\section{Normal appearances}

$T_{1}$ dependent images display high contrast between grey and white matter so that the normal process of myelination may be observed in vivo (Fig. 1). ${ }^{5}$ From necropsy studies it is known that there is a rapid phase of myelination in the first 2 years of life followed by a slower phase extending into the second decade. NMR appearances correspond well with these findings. The ventricular system shows clearly and in neonates dark (long $T_{1}$ ) areas in the peri-

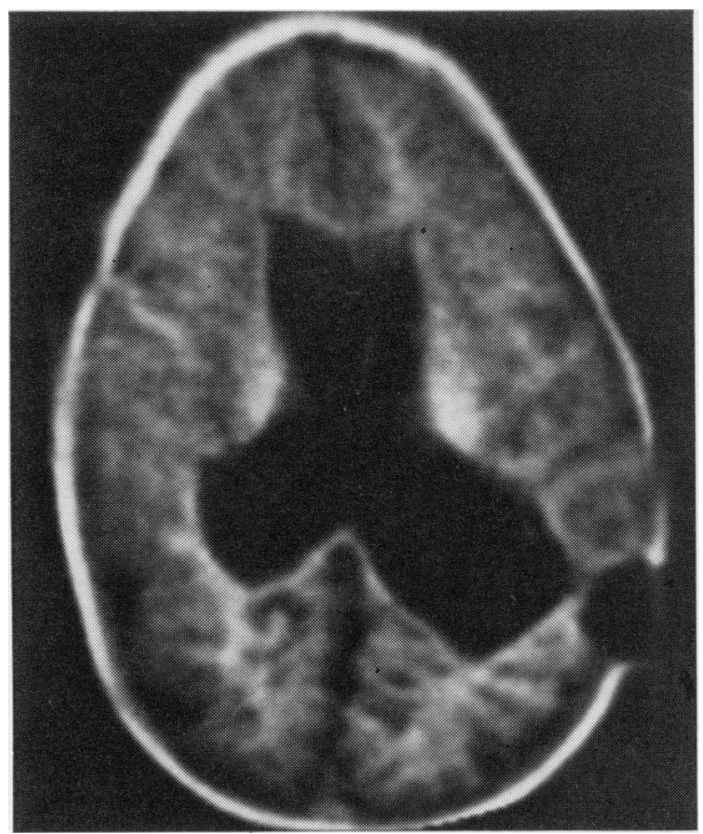

Fig. $2 T_{1}$ dependent scan, showing hydrocephalus with delayed myelination in an infant aged 30 months.

The ventricular system is dilated and the degree of myelination is less than in the normal 20 month child seen in figure $1 b$. A ventricular shunt artefact is noted posterolaterally. ventricular regions are seen in a similar manner to CT. Cortical bone has a low proton density and gives very little signal.

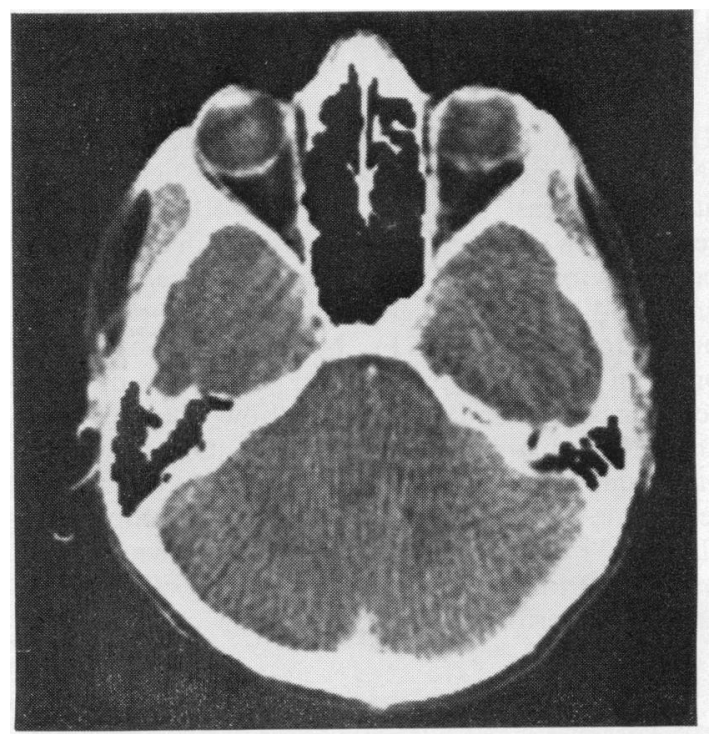

(a)

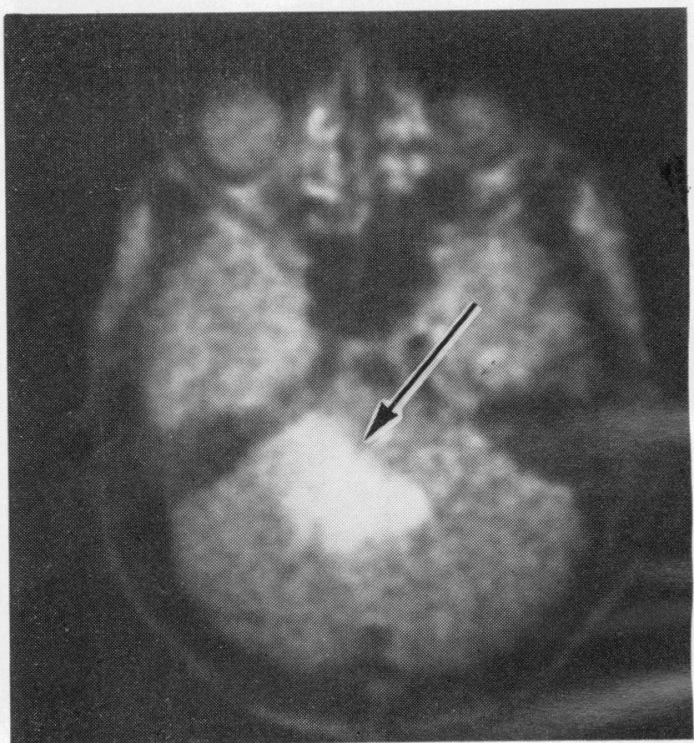

(b)

Fig. 3 Enhanced CT scan (a) and $T_{2}$ dependent scan $(b)$ of the same intrinsic tumour of the brainstem in a child aged 12 years. The tumour is poorly shown with CT (a) but the white mass is highlighted (arrow) on (b). 


\section{Abnormal appearances}

Delays in myelination can be recognised with NMR (Fig. 2) and have been seen after intraventricular haemorrhage and probable rubella infection. Many conditions, such as intracerebral haemorrhage, infarction, hydrocephalus, and cystic change, shown by ultrasound and CT may also be seen with NMR and some, such as periventricular oedema and leucodystrophy, show more clearly with NMR.

Cerebral tumours have been identified with NMR and the technique is valuable in distinguishing intrinsic from extrinsic tumours. Tumours have also been shown in the posterior fossa, when only minimal change has been evident with CT (Fig. 3). Abnormalities have been seen in patients with Hurler's syndrome, cerebral palsy, and other conditions using NMR, but the importance of these is uncertain.

\section{Conclusion}

So far only about 70 children world wide have been studied with NMR, and much more work is necessary to establish its role in clinical practice. Many questions about the design of the NMR machine remain unanswered. For example, the static magnet can be produced by permanent, resistive, or cryogenic magnets, each of which has advantages and disadvantages in cost and performance. Static magnetic field strengths in present use vary between 400 and 15000 Gauss and there is little agreement about the best level for routine clinical use. There are also many applications of NMR, such as the use of paramagnetic contrast agents, ${ }^{23} \mathrm{Na}$ imaging, flow studies, and real time imaging, which have been shown in animals but have yet to be evaluated in clinical practice.

Until further clinical studies are completed and the many options in machine design and imaging technique are evaluated, the exact role of NMR imaging in relation to other imaging techniques will remain uncertain. Nevertheless, the initial results suggest that this technique will be a source of considerable interest and value over the next few years.

\section{References}

1 Bailes D R, Young I R, Thomas D J, Straughan K, Bydder G M, Steiner R E. NMR imaging of the brain using spin-echo sequences. Clin Radiol 1982; 33: 395-414.

2 Bydder G M, Steiner R E, Young I R, et al. Clinical NMR imaging of the brain: 140 cases. AJR 1982; 139: 216-36.

3 Budinger T F. Nuclear magnetic resonance (NMR) in vivo studies: known thresholds for health effects. J Comp Assist Tomogr 1981 ; 5: 800-11.

4 Saunders R D, Orr J S. Biologic effects of NMR. In: Partain C L, James A E, Rollo F D, Price R R, eds. NMR imaging. Philadelphia: Saunders, 1983: 383-96.

5 Levene M I, Whitelaw A, Dubowitz V, et al. Nuclear magnetic resonance imaging of the brain in children. Br Med J 1982; 285: 774-6.

G M Bydder AND A Whitelaw Departments of Diagnostic Radiology and Paediatrics, Royal Postgraduate Medical School, Hammersmith Hospital, Du Cane Road, London W12 OHS 